\title{
A Mathematical Model to Control the Prevalence of a Directly and Indirectly Transmitted Disease
}

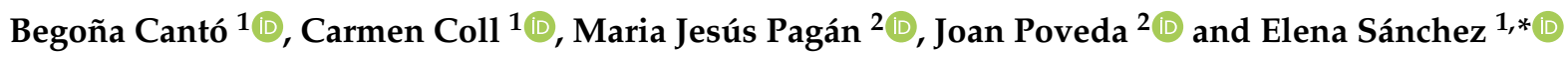 \\ 1 Institut de Matemàtica Multidisciplinar, Universitat Politècnica de València, 46071 València, Spain; \\ bcanto@mat.upv.es (B.C.); mccoll@mat.upv.es (C.C.) \\ 2 Grupo de Investigación e Innovación Alimentaria, Universitat Politècnica de València, 46071 València, Spain; \\ jpagan@tal.upv.es (M.J.P.); joapogi@alumni.upv.es (J.P.) \\ * Correspondence: esanchezj@mat.upv.es
}

check for updates

Citation: Cantó, B.; Coll, C.; Pagán M.J.; Poveda, J.; Sánchez, E. A Mathematical Model to Control the Prevalence of a Directly and Indirectly Transmitted Disease. Mathematics 2021, 9, 2562. https:// doi.org/10.3390/math9202562

Academic Editor: Mikhail Kolev

Received: 9 September 2021

Accepted: 7 October 2021

Published: 13 October 2021

Publisher's Note: MDPI stays neutral with regard to jurisdictional claims in published maps and institutional affiliations.

Copyright: (C) 2021 by the authors. Licensee MDPI, Basel, Switzerland. This article is an open access article distributed under the terms and conditions of the Creative Commons Attribution (CC BY) license (https:/ / creativecommons.org/licenses/by/ $4.0 /)$.

\begin{abstract}
In this paper, a mathematical model to describe the spread of an infectious disease on a farm is developed. To analyze the evolution of the infection, the direct transmission from infected individuals and the indirect transmission from the bacteria accumulated in the enclosure are considered. A threshold value of population is obtained to assure the extinction of the disease. When this size of population is exceeded, two control procedures to apply at each time are proposed. For each of them, a maximum number of steps without control and reducing the prevalence of disease is obtained. In addition, a criterion to choose between both procedures is established. Finally, the results are numerically simulated for a hypothetical outbreak on a farm.
\end{abstract}

Keywords: epidemic model; direct and indirect transmission; discrete-time system; stability; control

\section{Introduction}

Mathematical models are frequently used to analyze the behavior of an infectious disease on a group of individuals. The infectious agent that causes the disease can be transferred between an infected and a susceptible individual (direct transmission), from a reservoir where susceptible individuals are exposed to the pathogen (indirect transmission), or even through both types of transmission. Identifying the route of transmission is important as this facilitates better prevention system and control of infectious diseases. In the literature there are several works with direct transmission models such as [1-5] and indirect transmission [6-9]. However, the case in which the transmission is direct and indirect has not been studied. This contrasts markedly the behavior of most diseases such as salmonella, typhoid fever or COVID-19. Papers related to direct and indirect transmission are [10-12].

In this work, using theoretical models, we analyze the behavior of infectious diseases that can be transmitted directly and indirectly. In particular, we suggest a direct and indirect transmission model where the pathogen cannot be reproduced in the environment. We study the epidemiological dynamics at the population level and we also propose control techniques to ensure that the disease evolves favorably.

Unlike other papers that appear in the literature on models of direct or/and indirect disease transmission, the novelty of this work consists of the method followed to control the disease. In our case, we propose two different strategies that are applied periodically. In both cases, we obtain an N-periodic mathematical model. The main result is obtaining a threshold for each of these strategies. This provides us with the optimal time interval in which we can be without acting on the process and with the assurance that the disease tends to disappear. In addition, the information obtained can be used to compare the two strategies and determine which is the most appropriate for the different models that can be obtained from the parameters. 
Generally, when modeling epidemic processes, a discrete time approach has advantages over a model that uses differential equations because it is more realistic, since the epidemic statistics are made from given time intervals and not continuously (that is, significant for analyzing the rapid spread of epidemics). Examples of discrete epidemic models can be found in $[2,3,5-7,13-15]$ and references given there. In this case, the model is described by a non-linear system $z(t+1)=f(z(t))$ where the variables and parameters involved are the usual ones in this type of process. To study the evolution of the disease, equilibrium points are determined, disease-free and endemic equilibrium points. A linear model can arise from the linearization of the above system around one of them, and this approach can provide sufficient precision to describe the evolution of the system and specify whether or not the disease can be controlled.

The paper is organized as follows. In Section 2, the discrete-time non-linear mathematical epidemic model is shown. The stability of the equilibrium points is studied in terms of the size of the population in the enclosure. Our main results are given Section 3 where two control procedures to lead the infection towards disappearance are investigated. Moreover, in function of the survival rates, a criterion to determine the better one of them is established. In Section 4 the theoretical results are illustrated with some examples applying both strategies to herds of several population sizes, and the conclusions follow in Section 5 .

\section{Mathematical Model and Problem Statement}

In this chapter we propose a mathematical model to describe the behavior of a disease in a farm. This model can obtain the prevalence of infection depending of the size of the population in the farm. The animals can be infected through direct or indirect transmission. So, in this model we have opted for these two approaches.

We consider a transmission model consisting of two compartments: susceptible $(S(t))$, infected $(I(t))$, and in addition to the susceptible and infected individuals variables is considered a variable measuring the amount of contaminant, pathogen or bacteria, $(B(t))$, that is found in the environment which has been generated by infected individuals.

In the model of spread of infectious disease we consider that replaced individuals are introduced into the susceptible class and after infection the individuals transfer to the infected class. It is assumed that only infected individuals produce bacteria $(B(t))$ and infected individuals remain in the enclosure together. Moreover, at each stage the cage is closed and only the dead animals are replaced. That is, the size of the population always remains constant $P=S(t)+I(t)$ with replacement of dead individuals.

Considering all the above assumptions, we can represent our model (Figure 1).

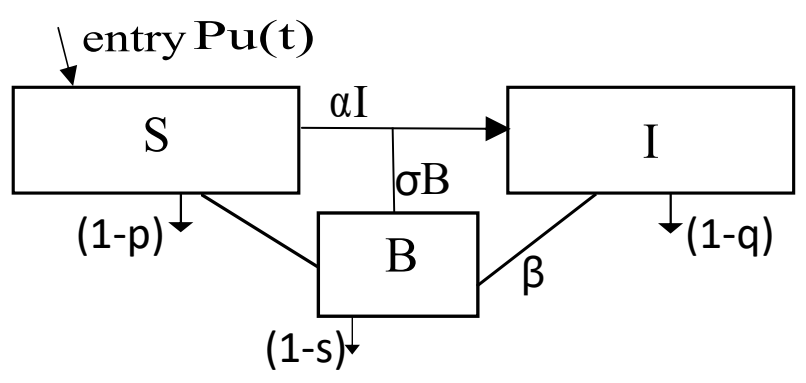

Figure 1. SIB model.

The Table 1 contains the list of parameters, symbols and abbreviations used in the paper. 
Table 1. Parameters, symbols and abbreviations in the paper.

\begin{tabular}{ll}
\hline$P$ & Size of population. \\
$S(t)$ & Susceptible population at time $t$. \\
$I(t)$ & Infected population at time $t$. \\
$B(t)$ & Amount of bacteria at time $t$. \\
$\alpha(0<\alpha<1)$ & Direct transmission rate. \\
$\sigma(0<\sigma<1)$ & Indirect transmission rate. \\
$u(t)$ & Replacement rate of the population \\
& $(u(t) P$ dead individuals at time $t)$. \\
$p(0<p<1)$ & Survival rate of the susceptible population. \\
$q(0<q<1)$ & Survival rate of the infected population. \\
$S(0<s<1)$ & Survival rate of the bacteria. \\
$\beta(\beta>0)$ & Amount of bacteria produced by infected individual. \\
$\lambda$ & eigenvalue of a matrix. \\
$\rho(M)$ & spectral radius of $M$ (maximum modulus of its eigenvalues). \\
$x_{f}$ & DFEP, disease-free equilibrium point. \\
$x_{e}$ & EEP, endemic equilibrium point. \\
$S_{e}$ & Size of susceptible population in endemic equilibrium point. \\
$N$ & period of time. \\
$M_{s}=\Phi_{M}(s+N, s)$ & Monodromy matrix. \\
$N_{a}^{\star}$ & Threshold value such that the infectious disease dissappears \\
& because the infected individuals are removed. \\
$N_{b}^{\star}$ & Threshold value such that the infectious disease dissappears \\
& because the enclosure is disinfected. \\
\hline
\end{tabular}

From the parameters shown in Table 1 and denoting

$$
x(t)=\left(x_{i}(t)\right)_{i=1,2,3}^{T}=(S(t), I(t), B(t))^{T},
$$

the dynamics of the disease are modeled using the following system of difference equations

$$
x(t+1)=\bar{A} x(t)+F(x(t))+P u(t) e_{1}, \quad t \geq 0
$$

with

$$
\begin{gathered}
\bar{A}=\left(\begin{array}{ccc}
p & 0 & 0 \\
0 & q & 0 \\
0 & \beta & s
\end{array}\right) \\
F(x(t))=\left(\alpha x_{1}(t) x_{2}(t)+\sigma x_{1}(t) x_{3}(t)\right)\left(\begin{array}{r}
-1 \\
1 \\
0
\end{array}\right)
\end{gathered}
$$

and $e_{1}=\left(\begin{array}{l}1 \\ 0 \\ 0\end{array}\right)$. Assuming $x_{1}(t)+x_{2}(t)=P, \forall t \geq 0$, the replacement of individuals satisfies $u(t) P=(-p+q) x_{1}(t)+(1-q) x_{2}(t)$ and we obtain

$$
x(t+1)=A x(t)+F(x(t))+(1-q) P e_{1}, \quad t \geq 0,
$$

with

$$
A=\left(\begin{array}{lll}
q & 0 & 0 \\
0 & q & 0 \\
0 & \beta & s
\end{array}\right)
$$

This mathematical model representing the dynamics of a direct and indirect infection on the farm is a nonlinear discrete-time system whose equilibrium points $\hat{x}, \hat{x}=A \hat{x}+$ $F(\hat{x})+B(1-q)$, are the disease-free equilibrium point (DFEP) $x_{f}=(P, 0,0)$ and the endemic equilibrium point $(\mathrm{EEP}) x_{e}=\left(S_{e}, P-S_{e},\left(P-S_{e}\right) \frac{\beta}{1-s}\right)$ with $S_{e}=\frac{(1-q)(1-s)}{\sigma \beta+\alpha(1-s)}$. 
If we analyze the behavior of the solution of the system around the equilibrium point $\hat{x}$ we have the following linear approach

$$
z(t+1)=\left(\begin{array}{rrr}
q-\sigma \hat{x}_{3}-\alpha \hat{x}_{2} & -\alpha \hat{x}_{1} & -\sigma \hat{x}_{1} \\
\sigma \hat{x}_{3}+\alpha \hat{x}_{2} & q+\alpha \hat{x}_{1} & \sigma \hat{x}_{1} \\
0 & \beta & s
\end{array}\right) z(t),
$$

where we have considered the translation $z(t)=x(t)-\hat{x}$ where $\hat{x}=\left(\hat{x}_{1}, \hat{x}_{3}, \hat{x}_{3}\right)$ is some equilibrium point. In Control Theory, it is known that the solution of an autonomous discrete-time linear system $x(t+1)=M x(t)$ is asymptotically stable to 0 if and only if the state matrix $M$ is stable. That is, $\rho(M)<1$, with $\rho(M)$ denoting the spectral radius of $M$ (maximum modulus of its eigenvalues). Calculating the eigenvalues of the state matrix in (6), these are $q$ and

$$
\frac{1}{2}\left(q+\alpha\left(\hat{x}_{1}-\hat{x}_{2}\right)-\sigma \hat{x}_{3}+s \pm \sqrt{\left(q+\alpha\left(\hat{x}_{1}-\hat{x}_{2}\right)-\sigma \hat{x}_{3}-s\right)^{2}+4 \beta \sigma \hat{x}_{1}}\right),
$$

Analyzing the solution of this system for each one of the equilibrium points, we have the following results.

When we consider the behavior of the system via linear approximation around the disease-free equilibrium point (DFEP) $x_{f}$, we have

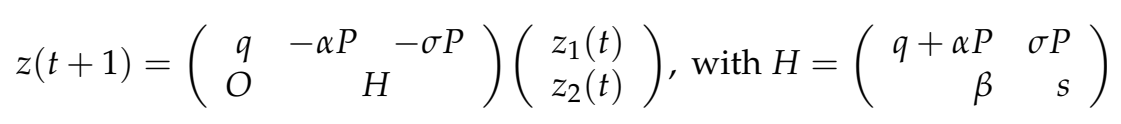

where we have considered the translation $z(t)=\left(z_{1}(t) z_{2}(t)\right)^{T}=x(t)-x_{f}$ with $z_{1}(t)=x_{1}(t)-P$ and $z_{2}(t)=\left(x_{2}(t) x_{3}(t)\right)^{T}$.

The capacity of dissemination or spread of the infection is quantified through the eigenvalues of the state matrix $q$ and the eigenvalues of $H$ given by (7), in this particular case

$$
\lambda_{1,2}=\frac{q+\alpha P+s \pm \sqrt{(q+\alpha P-s)^{2}+4 \sigma \beta P}}{2}
$$

Spectral radius of matrix $H$ indicates the asymptotic stability to zero $(\rho(H)<1)$ or not $(\rho(H) \geq 1)$ of the linear system, and consequently the asymptotic stability or not of the dynamic process to disease-free equilibrium point. By (9), we prove that $\lambda_{1}=\rho(H)<1$ if and only if the size of the population $P$ is less than $S_{e}=\frac{(1-q)(1-s)}{\sigma \beta+(1-s) \alpha}$ :

$$
\begin{aligned}
\lambda_{1}<1 & \leftrightarrow(q+\alpha P-s)^{2}+4 \sigma \beta P<(2-(q+\alpha P+s))^{2} \\
& \leftrightarrow-2 s(q+\alpha P)+4 \sigma \beta P<4-4(q+\alpha P+s)+2 s(q+\alpha P) \\
& \leftrightarrow \sigma \beta(\alpha-s \alpha+\sigma \beta) P<1-q-s+q s \\
& \leftrightarrow P<\frac{(1-q)(1-s)}{\sigma \beta+\alpha(1-s)}=S_{e} .
\end{aligned}
$$

If we analyze the behavior of the solution of the system around the endemic equilibrium point (EEP) $x_{e}$ we have the linear approach given in (6) and the eigenvalues of the state matrix are $q$ and $\lambda_{1,2}(\hat{x})$ given in (7) when the equilibrium point is $\hat{x}=x_{e}$. Using $x_{e}=\left(S_{e}, P-S_{e},\left(P-S_{e}\right) \frac{\beta}{1-s}\right)$ with $S_{e}=\frac{(1-q)(1-s)}{\sigma \beta+\alpha(1-s)}$ we can prove that the spectral radius of the state matrix, $\lambda_{1}$, given in (7) is less than 1 if and only if the size of the population $P$ is greater than $S_{e}$.

We have obtain the following result. 
Theorem 1. Consider the system given by (1)-(3). If the size of population $P$ is less than $S_{e}=\frac{(1-q)(1-s)}{\sigma \beta+\alpha(1-s)}$, then it is asymptotically stable to disease-free equilibrium point. If $P>S_{e}$, then it is asymptotically stable to endemic equilibrium point.

Note that, in the case $P=S_{e}$, the system is not asymptotically stable to disease-free equilibrium point and it is a critical case which is included in our study. When the size of population is greater than or equal to the threshold value $S_{e},(\rho(H) \geq 1)$, the instability of the system around the disease-free equilibrium point leads to the disease remaining in the farm. Then, some interventions are necessary to control the spread of the disease. Next, we establish our aim objective of this work.

Problem statement. If $P \geq S_{e},(\rho(H) \geq 1)$, the infected population does not disappear and it remains endemic. Some strategies are necessary to make extinct the infection. Our goal is to analyze how often we should act on the process to ensure that the infection does not spread and tends to be eradicated in the enclosure. As the infected individuals and bacteria are the focus of infection, we propose to act periodically, that is, follow each $N$ steps: (a) Removing the infected individuals or (b) removing the bacteria in the enclosure, through periodic programs.

This problem is studied in the following section.

\section{Periodic Control Leading the Infection to Extinction}

We consider the system given by (1)-(3) with $P \geq S_{e}$. Then, the system is not stable to disease-free equilibrium point and we want to redirect the solution of the system towards this point using the mentioned techniques in the problem statement. In both detection and disinfection processes, we consider an $N$-periodic treatment. Our study led us to use of the Control Theory of $\mathrm{N}$-periodic linear discrete-time system, see [2,16]. We construct a model to analyze each case:

(a) An N-periodic model representing the periodic detection and elimination of infected individuals. Given $N$, when the infected individuals are removed, at time $t=k N$, $k \geq 0$ the survival rate of it, the direct transmission rate and production of bacteria are considered zero. In the rest of the steps, these parameters take their estimated value. Thus, the parameters $\alpha, q$ and $\beta$ becoming $N$-periodic: $\alpha(t+N)=\alpha(t)=$ $\{0, t=0 ; \alpha, 1 \leq t<N\}, q(t+N)=q(t)=\{0, t=0 ; q, 1 \leq t<N\}$ and $\beta(t+N)=\beta(t)=\{0, t=0 ; \beta, 1 \leq t<N\}$. The $N$-periodic system is given by

$$
\begin{array}{ll} 
& z_{2}(t+1)=H_{a}(t) z_{2}(t) \\
\text { with } \quad H_{a}(0)=\left(\begin{array}{cc}
0 & \sigma P \\
0 & s
\end{array}\right), \quad H_{a}(t)=H, t=1, \ldots, N-1,
\end{array}
$$

$H_{a}(t+N)=H_{a}(t), t \geq 0$, with $z_{2}(t)=\left(x_{2}(t) x_{3}(t)\right)^{T}$ defined as under equation (8). Moreover, in this case, at time 0 , the infected individuals are removed, then $x_{2}(0)=0$, but bacteria may be in the enclosure, then $x_{3}(0)=x_{3}^{0}$.

Now, infection can occur in both ways, directly through contact with an infected individual or through contact with the bacteria, and we also propose two different actions and make a comparison between them. We give conditions to determine, in each case, which of the two techniques is the most effective. In this way, the second strategy is:

(b) An N-periodic disinfection of the amount of bacteria in the enclosure. Then, the indirect transmission rate and the survival rate of the bacteria are considered zero at steps multiple of $N$. That is, the parameters $s$ and $\sigma$ are replaced by two $N$-periodic parameters $\sigma(t)$ and $s(t)$ which are equal to $s$ and $\sigma$ at all times except at multiple of $N$ times: $s(t+N)=s(t)=\{0, t=0 ; s, 1 \leq t<N\}$ and $\sigma(t+N)=\sigma(t)=$ $\{0, t=0 ; \sigma, 1 \leq t<N\}$. A first application of this technique was introduced in some previous works on Salmonella infection in a hen house only considering transmission 
through indirect contact, $[7,17]$. Now, the new $\mathrm{N}$-periodic system with direct and indirect transmission is given by

$$
\begin{gathered}
z_{2}(t+1)=H_{b}(t) z_{2}(t) \\
\text { with } \quad H_{b}(0)=\left(\begin{array}{cc}
q+\alpha P & 0 \\
\beta & 0
\end{array}\right) \quad H_{b}(t)=H, t=1, \ldots, N-1,
\end{gathered}
$$

$H_{b}(t+N)=H_{b}(t), t \geq 0$, with $z_{2}(t)=\left(x_{2}(t) x_{3}(t)\right)^{T}$ defined as under Equation (8).

Now, the disinfecting of the enclosure is made at time 0 , then $x_{3}(0)=0$, but infected individuals may be in the enclosure, then $x_{2}(0)=x_{2}^{0}$.

It is known that to analyze the stability of a periodic system it is necessary to work with the monodromy matrix, which is the ordered product of all the coefficient matrices involved in the periodic process during a period of time, see for instance $[2,16]$ and the references therein. Remember that the asymptotic stability to 0 of an $\mathrm{N}$-periodic discretetime linear system $x(t+1)=M(t) x(t), M(t+N)=M(t), t \geq 0$ is characterized by $\rho\left(\Phi_{M}(k+N, k)\right)<1,0 \leq k<N$, being $\Phi_{M}(k+N, k)=M(k+N-1) \ldots M(k)$ the monodromy matrix at time $k$. Moreover, these spectral radius are equals for all $k$, see [16]. Thus, it is sufficient to study the stability of $\Phi_{M}(N, 0)$.

Now we are going to expand on the analysis of the control techniques proposed. In our cases $(a)$ and $(b)$, the monodromy matrix is $H_{k}^{a}=\Phi_{H_{a}}(k+N, k)=H_{a}(k+N-1) \cdots H_{a}(k)$, $H_{k}^{b}=\Phi_{H_{b}}(k+N, k)=H_{b}(k+N-1) \cdots H_{b}(k), 0 \leq k \leq N-1$, respectively. Additionally, the system (10), (11), is asymptotically stable to zero if $\rho\left(H_{0}^{a}\right)<1, \rho\left(H_{0}^{b}\right)<1$, respectively.

In our model, the effect of direct transmission and indirect transmission is reflected in the parameters that appear in the coefficient matrix of the system. This information is used to obtain the monodromy matrix of the periodic system that we use to study the behavior of the model. Therefore, we have to study the stability of the monodromy matrix in $k=0$. In the following results we have considered the two control strategies that we discussed at the beginning of this section. This study will provide us with the threshold values for the period $N$ that ensure the stability of the corresponding $N$-periodic system. We denote by $N_{a}^{\star}$ the bound for the control action $(a)$ and by $N_{b}^{\star}$ the bound for the control action $(b)$. The way to obtain both bounds is analogous and is made explicit in the proof of the following result. Moreover, in the theorem, the condition $q+\alpha P<1$ is needed to show that $j_{b}(N)$ given in (16) is a decreasing function.

Theorem 2. Consider an epidemic outbreak modeled by system (1)-(3). If $q+\alpha P<1$ and the eigenvalues given in (9) satisfy $0<\lambda_{2}<1 \leq \lambda_{1}$, then there exist threshold values $N_{a}^{\star}$ and $N_{b}^{\star}$ such that the infectious disease disappears when either the infected individuals are removed each $N_{a}^{\star}$ steps or the enclosure is disinfected each $N_{b}^{\star}$ steps, respectively.

Proof. We develop the case (a) and in a similar way we can establish the results corresponding to the case $(b)$.

(a) In this case, we consider $H_{0}^{a}=\Phi_{H_{a}}(N, 0)$. From (10), we can calculate this monodromy matrix

$$
H_{0}^{a}=\Phi_{H_{a}}(N, 0)=H^{N-1} H_{a}(0)=\left(\begin{array}{cc}
0 & \star \\
0 & a(N)
\end{array}\right),
$$

and we obtain that its spectral radius is given by

$$
\rho\left(H_{0}^{a}\right)=a(N)=\frac{\lambda_{1}^{N}\left(s-\lambda_{2}\right)+\lambda_{2}^{N}\left(\lambda_{1}-s\right)}{\lambda_{1}-\lambda_{2}},
$$

where $\lambda_{1}$ and $\lambda_{2}$ are the eigenvalues of $H$ given in (9).

Our main goal is to find the maximum interval of time $N_{a}^{\star}$, so that $a\left(N_{a}^{\star}\right)<1<$ $a\left(N_{a}^{\star}+1\right)$. This value $N_{a}^{\star}$ will be the maximum number of steps maintaining stabilized the solution of the system, and hence, the disease evolves towards disappearance. For that, we 
construct the following functions of the period $N$ and the analysis of their behavior will lead us to ensure the existence of this point $N_{a}^{\star}$ :

$$
\begin{aligned}
& \Theta(N)=\left(\frac{\lambda_{2}}{\lambda_{1}}\right)^{N} \\
& j_{a}(N)=\lambda_{1}^{-N}\left(\frac{\lambda_{1}-\lambda_{2}}{\lambda_{1}-s}\right)+\frac{\lambda_{2}-s}{\lambda_{1}-s}
\end{aligned}
$$

From (12)-(14), we have that

$$
\begin{aligned}
& (a(N)-1) \frac{\lambda_{1}-\lambda_{2}}{\lambda_{1}^{N}\left(\lambda_{1}-s\right)}= \\
& =\left(\frac{\lambda_{1}^{N}\left(s-\lambda_{2}\right)+\lambda_{2}^{N}\left(\lambda_{1}-s\right)}{\lambda_{1}-\lambda_{2}}-1\right) \frac{\lambda_{1}-\lambda_{2}}{\lambda_{1}^{N}\left(\lambda_{1}-s\right)} \\
& =\underbrace{\frac{\lambda_{2}^{N}}{\lambda_{1}^{N}}}_{\Theta(N)}-\underbrace{\frac{\left(\lambda_{1}-\lambda_{2}\right)+\lambda_{1}^{N}\left(\lambda_{2}-s\right)}{\lambda_{1}^{N}\left(\lambda_{1}-s\right)}}_{j_{a}(N)} \\
& =\Theta(N)-j_{a}(N) \text {. }
\end{aligned}
$$

Now, it is important make the following observation. From an unstable initial epidemiological process, we have $\lambda_{2}<\lambda_{1}=\rho(H)$ and $0<s<1 \leq \lambda_{1}$. If, then, we assume $\lambda_{2}>0$, these conditions lead us to functions $\Theta(N)$ and $j_{a}(N)$ given in (13) and (14) are decrease functions and $\Theta(1)<j_{a}(1)$.

Moreover, we have that

$$
\lim _{N \rightarrow \infty} j_{a}(N)=\frac{\lambda_{2}-s}{\lambda_{1}-s}<0=\lim _{N \rightarrow \infty} \Theta(N) .
$$

Then, we can assure that the order relation between $\Theta(N)$ and $j_{a}(N)$ changes for some values of $N$. That is, there exists $N_{a}^{\star}$ such that

$$
\begin{array}{lll}
\Theta\left(N_{a}^{\star}\right)-j\left(N_{a}^{\star}\right)<0 & \Rightarrow & a\left(N_{a}^{\star}\right)<1 \\
\Theta\left(N_{a}^{\star}+1\right)-j\left(N_{a}^{\star}+1\right)>0 & \Rightarrow & a\left(N_{a}^{\star}+1\right)>1 .
\end{array}
$$

In a similar way, we can analyze the case (b).

(b) Consider the $\mathrm{N}$-periodic model previously described in (11). Studying the spectral radius of the matrix of monodromy in $s=0$, we have to $H_{0}^{b}=\Phi_{H_{b}}(N, 0)=H^{N-1} H_{b}(0)=$ $\left(\begin{array}{cc}b(N) & 0 \\ \star & 0\end{array}\right)$ and

$$
\rho\left(H_{0}^{b}\right)=b(N)=\frac{\lambda_{1}^{N}\left(q+\alpha P-\lambda_{2}\right)+\lambda_{2}^{N}\left(\lambda_{1}-q-\alpha P\right)}{\lambda_{1}-\lambda_{2}} .
$$

Our next step is to find a period $N_{B}^{\star}$ in which we do not apply the control action but $b\left(N_{b}^{\star}\right)<1<b\left(N_{b}^{\star}+1\right)$. For that, we construct the following function

$$
j_{b}(N)=\lambda_{1}^{-N}\left(\frac{\lambda_{1}-\lambda_{2}}{\lambda_{1}-(q+\alpha P)}\right)+\frac{\lambda_{2}-(q+\alpha P)}{\lambda_{1}-(q+\alpha P)} .
$$

By (13)-(15)-(16), we have that $\left(b_{N}-1\right) \frac{\lambda_{1}-\lambda_{2}}{\lambda_{1}^{N}\left(\lambda_{1}-(q+\alpha P)\right)}=\Theta(N)-j_{b}(N)$. Assuming $0<q+\alpha P<1$ and $\lambda_{2}>0$, and using $\lambda_{1}=\rho(H) \geq 1$, we have that functions $\Theta(N)$ 
and $j_{b}(N)$ given in (13)-(16) are decrease functions, $\Theta(1)<j_{b}(1)$ and $\lim _{N \rightarrow \infty} j_{b}(N)=$ $\frac{\lambda_{2}-(q+\alpha P)}{\lambda_{1}-(q+\alpha P)}<0=\lim _{N \rightarrow \infty} \Theta(N)$. Hence, there exists $N_{b}^{\star}$ such that

$$
\begin{array}{lll}
\Theta\left(N_{b}^{\star}\right)-j\left(N_{b}^{\star}\right)<0 & \Rightarrow & b\left(N_{b}^{\star}\right)<1 \\
\Theta\left(N_{b}^{\star}+1\right)-j\left(N_{b}^{\star}+1\right)>0 & \Rightarrow & b\left(N_{b}^{\star}+1\right)>1 .
\end{array}
$$

Remark 1. The results of the theorem are valid under condition $q+\alpha P<1$. However, we will see late that using the survival rate $(0<s<1)$ as bound of $q+\alpha P$ will allow us to determine the control strategy to use.

The imposed conditions on the parameters lead us to restrictions on the size of the population $P$. First, the instability condition $\lambda_{1} \geq 1$ it is equivalent to $P \geq S_{e}$, and the condition $\lambda_{2}>0$ is held up if and only if $P<\frac{q s}{\sigma \beta-s \alpha}$ (it is proved from the definition of $\lambda_{1,2}$ given in (9)). On the other hand, note that if $q, s>\frac{1}{2}$ (it is frequent in the case of infectious disease as Salmonella, Brucella, Yersinia), then $S_{e}<\frac{q s}{\sigma \beta-s \alpha}$.

Based on these comments, from now on we rewritten the hypothesis of the above Theorem where the condition on $P, q+\alpha P<1$, is also considered.

Theorem 3. Consider an epidemic outbreak modeled by system (1)-(3) with $q, s>\frac{1}{2}$ and population size satisfying

$$
S_{e}<P<\min \left\{\frac{q s}{\sigma \beta-s \alpha}, \frac{1-q}{\alpha}\right\} .
$$

Then, there exist threshold values $N_{a}^{\star}$ and $N_{b}^{\star}$ such that the infectious disease disappears when either the infected individuals are removed each $N_{a}^{\star}$ steps or the enclosure is disinfected each $N_{b}^{\star}$ steps, respectively.

Now, we propose a new analysis to compare between the control strategies and we establish a criterion to choose one or another option of the proposed control strategies $(a)$ and $(b)$. We compare the two functions $j_{a}(N)$ and $j_{b}(N)$ in order to establish when is better to use one action than other. That is, which is the greatest $N_{a}^{\star}$ or $N_{b}^{\star}$. The result is given in the following Theorem.

Theorem 4. Consider an epidemic outbreak modeled by system (1)-(3) with $q, s>\frac{1}{2}$ and population size satisfying condition (17). Then,

(i) If $P<\frac{s-q}{\alpha}$, then the option of disinfection of the enclosure is the optimal one since $N_{a}^{\star}<N_{b}^{\star}$;

(ii) If $P>\frac{s-q}{\alpha}$, it is better the elimination of infected individuals option since $N_{b}^{\star}<N_{a}^{\star}$;

(iii) When $P=\frac{s-q}{\alpha}$ both techniques are useful in the same degree, since $N_{b}^{\star}=N_{a}^{\star}$.

Proof. The question is: Which function $j_{a}(N)$ or $j_{b}(N)$ intersects with $\Theta(N)$ before? This will depend on who is greater if $q+\alpha P$ or $s$ since by definitions given in (14)-(16), 


$$
\begin{aligned}
j_{a}(N) & -j_{b}(N)= \\
= & \lambda_{1}^{-N}\left(\lambda_{1}-\lambda_{2}\right)\left(\frac{1}{\lambda_{1}-s}-\frac{1}{\lambda_{1}-(q+\alpha P)}\right) \\
& +\frac{\lambda_{2}-s}{\lambda_{1}-s}-\frac{\lambda_{2}-(q+\alpha P)}{\lambda_{1}-(q+\alpha P)} \\
= & \lambda_{1}^{-N}\left(\lambda_{1}-\lambda_{2}\right) \frac{s-(q+\alpha P)}{\left(\lambda_{1}-s\right)\left(\lambda_{1}-(q+\alpha P)\right)} \\
& +\frac{((q+\alpha P)-s)\left(\lambda_{1}-\lambda_{2}\right)}{\left(\lambda_{1}-s\right)\left(\lambda_{1}-(q+\alpha P)\right)} \\
= & \frac{((q+\alpha P)-s)\left(\lambda_{1}-\lambda_{2}\right)}{\left(\lambda_{1}-s\right)\left(\lambda_{1}-(q+\alpha P)\right)}\left(1-\lambda_{1}^{-N}\right) .
\end{aligned}
$$

Hence, if $q+\alpha P \leq s$, then $j_{a}(N) \leq j_{b}(N)$ and if $q+\alpha P \geq s$, then $j_{b}(N) \leq j_{a}(N)$.

Remark 2. In a farm it is common to use these strategies to keep an infectious outbreak under control, but the results optimizing the number of stages without acting on the process using deterministic models are unusual. The contribution of this work is precisely this, so the results are novel. Using the theory of periodic systems control on the epidemic mathematics model, we have been able to establish the greatest number of steps without applying one of the techniques with the infection tending to disappear. In addition, depending on the value of the parameters, we know which one optimizes the use of the farm's resources since it allows for more steps without applying the corresponding control strategy. From these results, we conclude when it is better to apply one control technique or another. The higher threshold value of $N$ allows us to perform more steps without acting on the process. Under the hypotheses of the Theorem 4, we can ensure which technique is optimal one, that is, the option allowing to take more steps without intervening.

\section{Control Model Simulation}

In this section we present a brief summary of how the use of these techniques has affected the dynamics of our model. In order to do this we simulate a hypothetical infection that is transmitted both by direct contact between susceptible and infected individuals, and indirectly through the spread of the contaminant or pathogen in the environment; in line with other modeling of communicable diseases such as salmonellosis, malaria, tuberculosis, brucellosis (see [18-24]).

In the simulation we have considered the parameter values corresponding to farm animals as follows: $\alpha=10^{-5}$-the direct transmission rate, $\sigma=10^{-6}$-the indirect transmission rate, $\beta=10^{3}-\mathrm{CFU}$ (colony-forming unit) the amount of bacteria production, and $s=0.8$ - the survival rate of the bacteria. We start from an unstable model, that is the spectral radius of the state matrix $H$ is greater than 1 . We have distinguished two scenarios corresponding to $q+\alpha P<s$ and to $q+\alpha P>s$ and for each one of them, we have made simulations analyzing the two control strategies presented in this work: (a) detection and elimination of the infected individuals and $(b)$ disinfection of the enclosure, in three enclosures with a size of population of 100, 200 and 400 individuals, respectively. (i) First scenario: Consider $q=0.7$ survival rate of infected individuals. Then $q+\alpha P<s$ if $P<10^{4}$. Note that the upper bounds to $P$ given in (17) are $\left\{\frac{q s}{\sigma \beta-s \alpha}, \frac{1-q}{\alpha}\right\}=\left\{564,3.10^{4}\right\}$. Thus, by Theorem 3 we consider size of the population $P<546$.

As $S_{e}=59$, the solution trajectory of the system is asymptotically stable to zero when the size of population is $P<59$. For instance, if $P=50$ we have $\lambda_{1}=0.9793$, but if $P>59, \lambda_{1}>1$. As illustrative example, in Figure 2 is shown the increase of the infected population when $P=100$. We observe that the solution trajectory tends to the endemic point $\left(S_{e}, I_{e}, B_{e}\right)=(59.8802,40.1198,200599)$. 


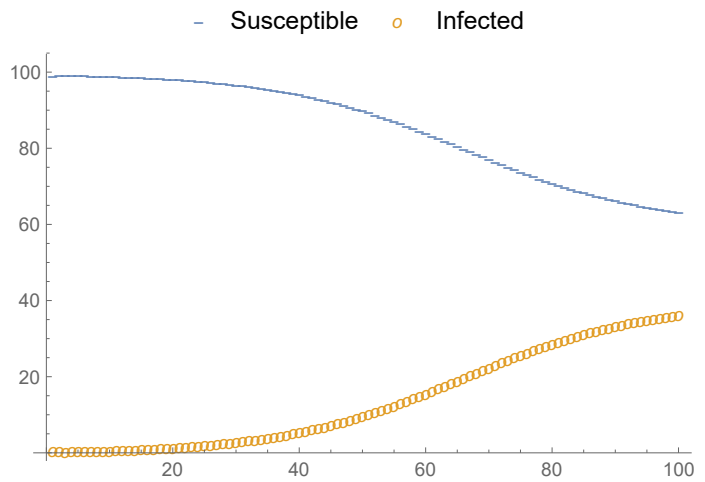

Figure 2. Evolution of the population of susceptible individuals $S(t)$ and infected individuals $I(t)$ when the population is $P=100 q=0.7$.

In Figure 3 is shown the graphics of the functions $\Theta(N), j_{I}(N), j_{B}(N)$ corresponding to the size of population $P=100,200$ and 400 .

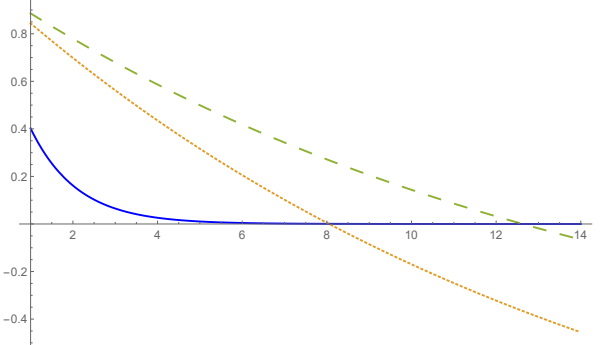

$P=100$

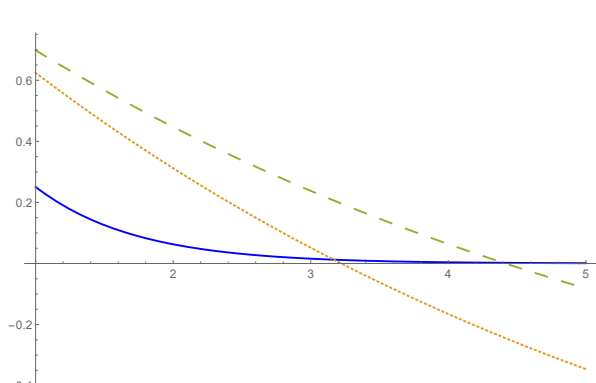

$P=200$

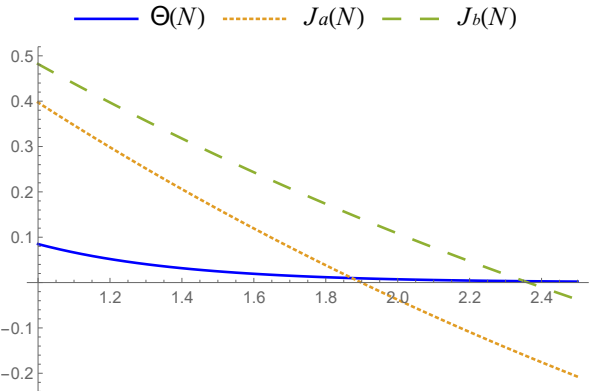

$P=400$

Figure 3. Intersection points between functions $J_{I}(N), J_{B}(N)$ and function $\Theta(N)$ when $q=0.7$.

These graphs show which method is the most indicated as a control action. All this is reflected in Table 2 where we can see the points of intersection between these functions. Using this information, we choose the control action corresponding to disinfection of the enclosure which confirms the results of the Theorem 4.

Table 2. Threshold times $N_{a}^{\star}$ and $N_{b}^{\star}$ to the model remain asymptotically stable to (DFEP) when $q=0.7$.

\begin{tabular}{cccc}
\hline & \multicolumn{3}{c}{ Population P } \\
\cline { 2 - 4 } & $\mathbf{1 0 0}$ & $\mathbf{2 0 0}$ & $\mathbf{4 0 0}$ \\
\hline$N_{a}^{\star}$ & 8 & 3 & 1 \\
$N_{b}^{\star}$ & 12 & 4 & 2 \\
\hline
\end{tabular}

(ii) Second scenario: Consider $q=0.9$ survival rate of infected individuals. Then $q+\alpha P>s$ for all $P$. By Theorem 3 we consider size of population $P<\min \left\{\frac{q s}{\sigma \beta-s \alpha}, \frac{1-q}{\alpha}\right\}=725$.

As $S_{e}=19$, the solution trajectory of the system is asymptotically stable to zero if $P<19$.

For instance, if $P=19$ we have $\lambda_{1}=0.9967$, but if $P>19$ we have $\lambda_{1}>1$. In Figure 4 is shown the increase of the infected population when $P=100$. In this case, the solution tends to the endemic point $\left(S_{e}, I_{e}, B_{e}\right)=(19.9601,80.0399,400200)$. 


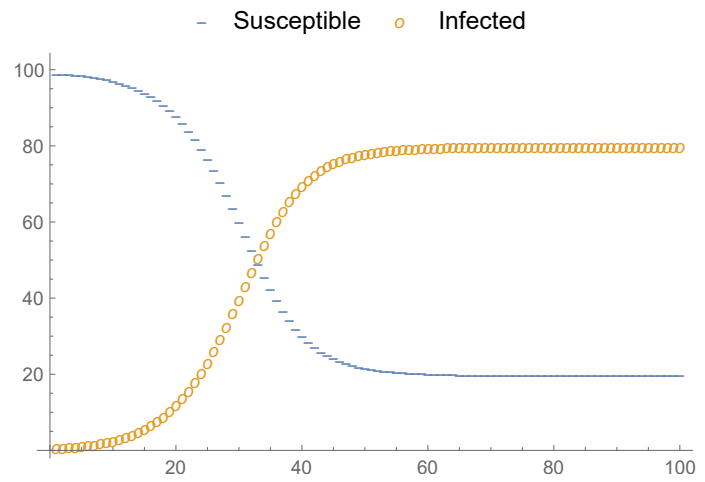

Figure 4. Evolution of the population of susceptible individuals $S(t)$ and infected individuals $I(t)$ when the population is $P=100$ and $q=0.9$.

In the Figure 5 is shown the graphics of the functions $\Theta(N), j_{I}(N), j_{B}(N)$ corresponding to the size of populations $P=100,200,400$. The points of intersection between these functions are given in Table 3. Using this information, we choose the control action corresponding to analysis and withdrawal of the infected individuals which confirms the results of Theorem 4.

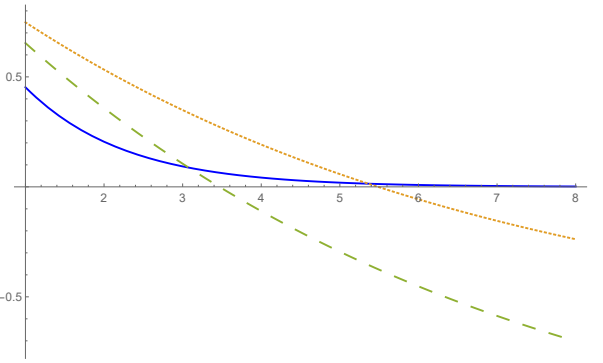

$P=100$

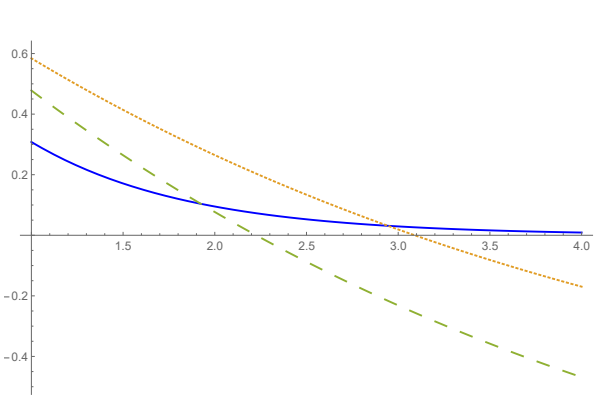

$P=200$

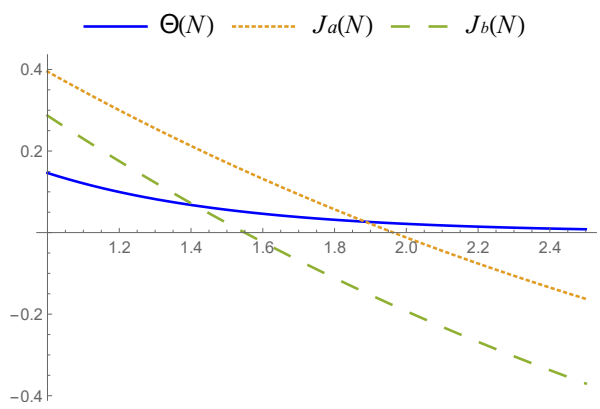

$P=400$

Figure 5. Intersection points between functions $J_{I}(N), J_{B}(N)$ and function $\Theta(N)$ when $q=0.9$.

Table 3. Threshold times $N_{I}^{\star}$ and $N_{B}^{\star}$ to the model remain asymptotically stable to (DFEP) when $q=0.9$.

\begin{tabular}{cccc}
\hline & \multicolumn{3}{c}{ Population P } \\
\cline { 2 - 4 } & $\mathbf{1 0 0}$ & $\mathbf{2 0 0}$ & $\mathbf{4 0 0}$ \\
\hline$N_{a}^{\star}$ & 5 & 2 & 1 \\
$N_{b}^{\star}$ & 3 & 1 & 1 \\
\hline
\end{tabular}

Note that when $q=0.7<s=0.8$ is most effective to eliminate the bacteria since the time interval without having to apply the disinfection control action is longer. However, when $q=0.9>s=0.8$ it is better to eliminate the infected individuals.

Below, in Tables 4 and 5, are shown the information about the spectral radius of the monodromy matrices $a_{N}$ from the elimination of infected individuals and $b_{N}$ of the disinfection, respectively. 
Table 4. Spectral radius of $\mathrm{N}$-periodic control model with elimination of infected individuals.

\begin{tabular}{lllllll}
\hline & \multicolumn{3}{c}{$\boldsymbol{a}_{\boldsymbol{N}}($ with $\boldsymbol{q}=\mathbf{0 . 7})$} & \multicolumn{3}{c}{$\boldsymbol{a}_{N}$ (with $\boldsymbol{q}=\mathbf{0 . 9 )}$} \\
\cline { 2 - 7 } & $\mathbf{P}=\mathbf{4 0 0}$ & $\mathbf{P}=\mathbf{2 0 0}$ & $\mathbf{P}=\mathbf{1 0 0}$ & $\mathbf{P}=\mathbf{4 0 0}$ & $\mathbf{P}=\mathbf{2 0 0}$ & $\mathbf{P}=\mathbf{1 0 0}$ \\
\hline$N=1$ & $\mathbf{0 . 8}$ & 0.8 & 0.8 & $\mathbf{0 . 8}$ & 0.8 & 0.8 \\
$N=2$ & 1.04 & 0.84 & 0.74 & 1.04 & $\mathbf{0 . 8 4}$ & 0.74 \\
$N=3$ & $\ldots$ & $\mathbf{0 . 9 7 2 4}$ & 0.748993 & $\ldots$ & 1.0124 & 0.7621 \\
$N=4$ & $\ldots$ & 1.1568 & 0.7729 & $\ldots$ & $\ldots$ & 0.8369 \\
$N=5$ & $\ldots$ & $\ldots$ & 0.8181 & $\ldots$ & $\ldots$ & $\mathbf{0 . 9 5 0 5}$ \\
$N=6$ & $\ldots$ & $\ldots$ & 0.8719 & $\ldots$ & $\ldots$ & 1.0972 \\
$N=7$ & $\ldots$ & $\ldots$ & 0.9317 & $\ldots$ & $\ldots$ & $\ldots$ \\
$N=8$ & $\ldots$ & $\ldots$ & $\mathbf{0 . 9 9 6 7}$ & $\ldots$ & $\ldots$ & $\ldots$ \\
$N=9$ & $\ldots$ & $\ldots$ & 1.0667 & $\ldots$ & $\ldots$ & $\ldots$ \\
\hline
\end{tabular}

Table 5. Spectral radius of $N$-periodic control model with disinfection of the enclosure.

\begin{tabular}{lllllll}
\hline & \multicolumn{3}{c}{$\boldsymbol{b}_{N}($ with $\boldsymbol{q}=\mathbf{0 . 7 )}$} & \multicolumn{3}{c}{$\boldsymbol{b}_{N}$ (with $\left.\boldsymbol{q}=\mathbf{0 . 9}\right)$} \\
\cline { 2 - 7 } & $\mathbf{P}=\mathbf{4 0 0}$ & $\mathbf{P}=\mathbf{2 0 0}$ & $\mathbf{P}=\mathbf{1 0 0}$ & $\mathbf{P}=\mathbf{4 0 0}$ & $\mathbf{P}=\mathbf{2 0 0}$ & $\mathbf{P}=\mathbf{1 0 0}$ \\
\hline$N=1$ & 0.704 & 0.702 & 0.701 & $\mathbf{0 . 9 0 4}$ & $\mathbf{0 . 9 0 2}$ & 0.901 \\
$N=2$ & $\mathbf{0 . 8 9 5 6}$ & 0.6928 & 0.5914 & 1.2172 & 1.0136 & 0.9118 \\
$N=3$ & 1.2321 & 0.7867 & 0.56467 & $\ldots$ & $\ldots$ & $\mathbf{0 . 9 9 1 6}$ \\
$N=4$ & $\ldots$ & $\mathbf{0 . 9 3 1 1}$ & 0.5750 & $\ldots$ & $\ldots$ & 1.12 \\
$N=5$ & $\ldots$ & $\ldots$ & 1.1141 & $\ldots$ & $\ldots$ & $\ldots$ \\
$N=6$ & $\ldots$ & $\ldots$ & 0.64 & $\ldots$ & $\ldots$ & $\ldots$ \\
$N=7$ & $\ldots$ & $\ldots$ & 0.6828 & $\ldots$ & $\ldots$ & $\ldots$ \\
$N=8$ & $\ldots$ & $\ldots$ & 0.73 & $\ldots$ & $\ldots$ & $\ldots$ \\
$N=9$ & $\ldots$ & $\ldots$ & 0.7811 & $\ldots$ & $\ldots$ & $\ldots$ \\
$N=10$ & $\ldots$ & $\ldots$ & 0.836 & $\ldots$ & $\ldots$ & $\ldots$ \\
$N=11$ & $\ldots$ & $\ldots$ & 0.895 & $\ldots$ & $\ldots$ & $\ldots$ \\
$N=12$ & $\ldots$ & $\ldots$ & $\mathbf{0 . 9 5 8 1}$ & $\ldots$ & $\ldots$ & $\ldots$ \\
$N=13$ & $\ldots$ & $\ldots$ & 1.0257 & $\ldots$ & $\ldots$ & $\ldots$ \\
\hline
\end{tabular}

Finally, focusing our attention in the case (i) First scenario, $q=0.7$ (analogously, we can analyze the case (ii) Second scenario, $q=0.9$ ), Figure 6 shows how the evolution of the population of infected and the number of bacteria on an enclosure with populations of size 100, 200 and 400 individuals, respectively.
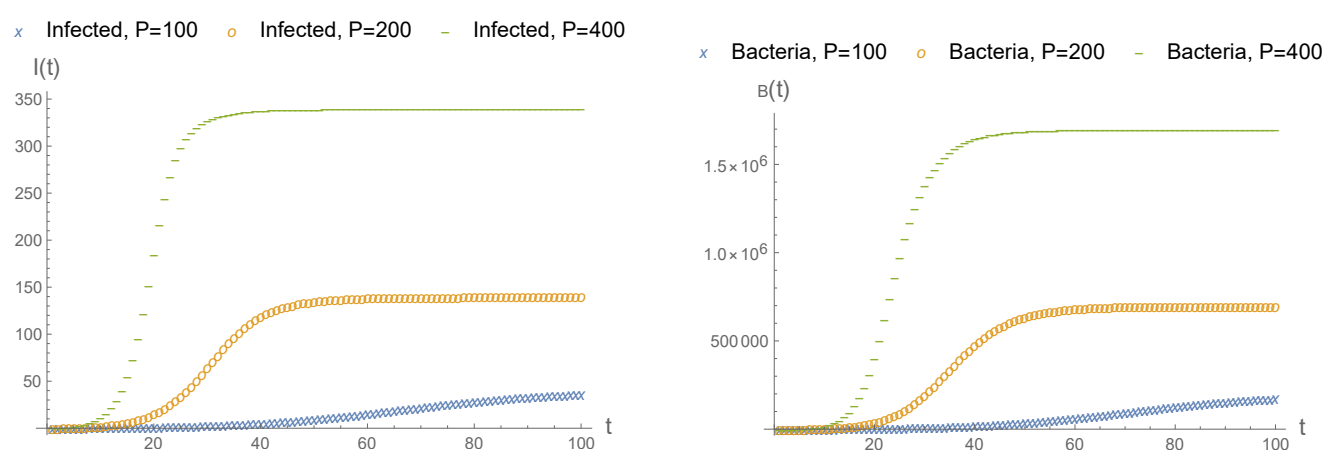

Figure 6. Evolution of the population of infected individuals $I(t)$ and bacteria $B(t)$ in the initial process, for a population of $P=100, P=200$ and $P=400$ individuals in the enclosure, when $q=0.7$.

When the size of the population is 100, the elimination of the accumulated bacteria is more effective than the elimination of those infected animals: With the strategy of case $(b)$ (disinfection of enclosure) we can spend 12 periods of time keeping the process without spreading (see Table 5), while by strategy of case (a) (eliminating the infected individuals), in step 8 the process becomes unstable spreading the infection (see Table 4). In Figure 7, 
we show the evolution of infected and bacteria variables under the two control strategies when the period is $N=9$.

For a population of 200 individuals, the process remains asymptotically stable up to time period 3 with the elimination of infected, but with the disinfection strategy we can act each 4 steps. It can be seen in the Figure 8 the evolution of the solution, corresponding to both strategies, when the period is $N=4$.

For a population of 400 individuals, it is necessary to remove the infected individuals at each stage in order to stabilize the process. However, the population of infected individuals and the bacteria tend to disappear if the disinfection action is made every two steps. This behavior is shown in Figure 9.
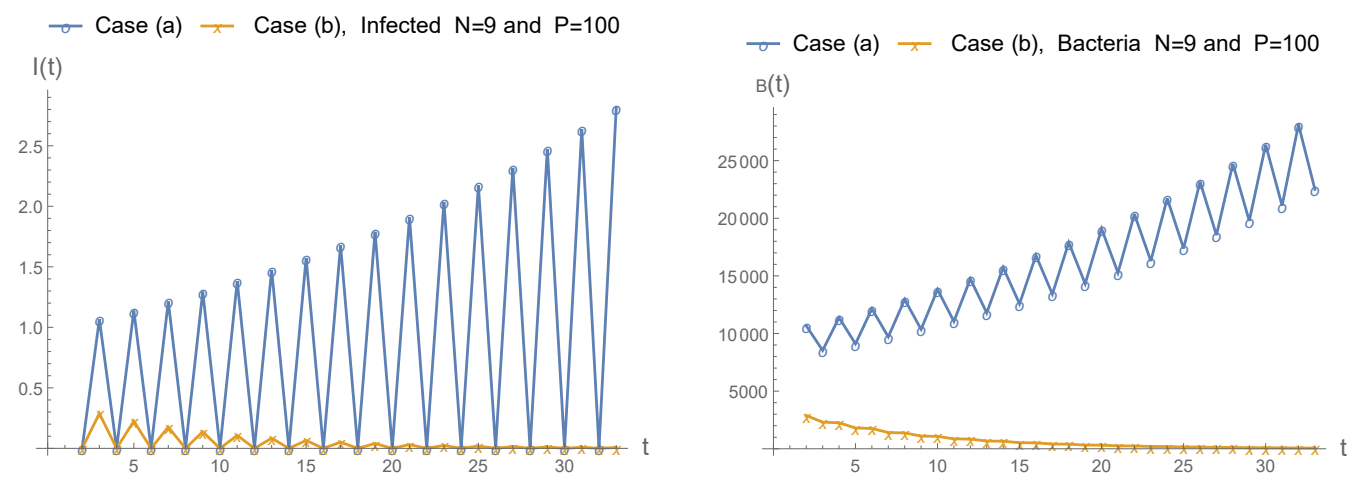

Figure 7. Evolution of the population of infected individuals $I(t)$ and bacteria $B(t)$ after applying the control strategy every 9 stages $(N=9)$, when the population $P=100$, when $q=0.7$.
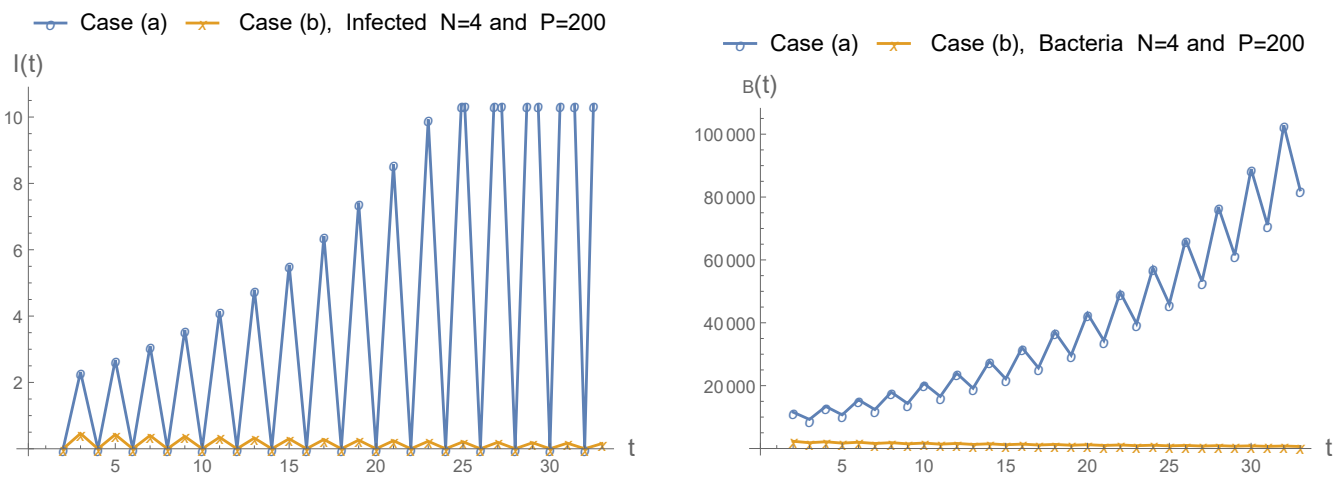

Figure 8. Evolution of the population of infected individuals $I(t)$ and bacteria $B(t)$ after applying the control strategy every 4 stages $(N=4)$, when the population $P=200$.
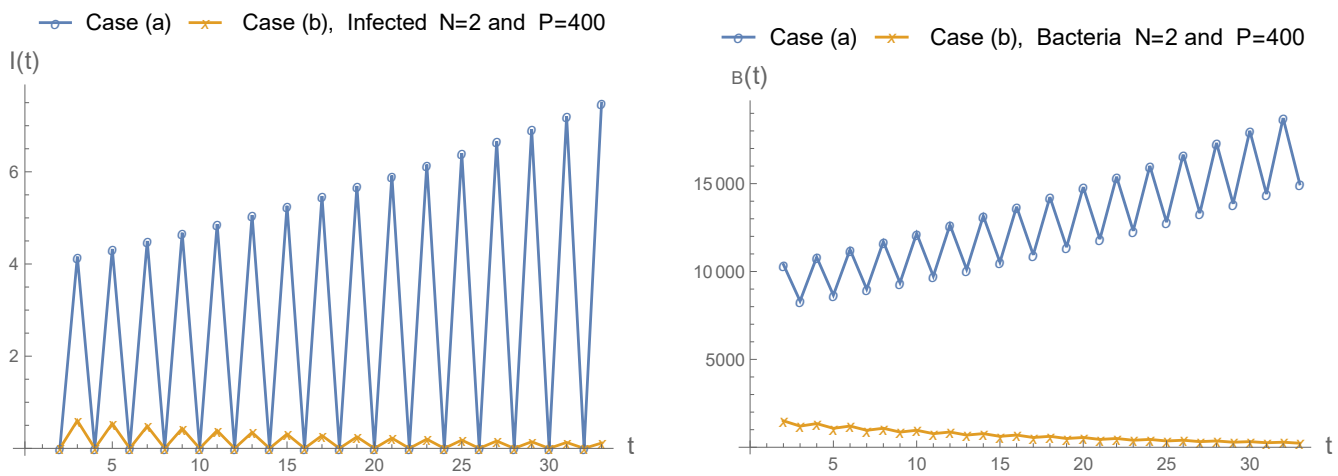

Figure 9. Evolution of the population of infected individuals $I(t)$ and bacteria $B(t)$ after applying the control strategy every 2 stages $(N=2)$, when the population $P=400$ when $q=0.7$. 


\section{Conclusions}

The spread of an infectious disease by direct and indirect transmission was considered. The process was mathematically represented by a non-linear discrete time system. Depending on the size of the population the disease tends to disappear, remains endemic or spreads, leading to a pandemic. After analyzing the different procedures that are usually used, two control strategies have been chosen: elimination of infected individuals and disinfection of the enclosure. The application of one of these procedures from time to time has led us to an N-periodic mathematical model. We have developed a method to find the optimal time interval without acting on the process and with the disease tending to disappear. We have obtained threshold points for each of the strategies and we use this information to compare the two strategies.

We can conclude that when the survival rate of infected individuals plus $\alpha P$ is lower than the survival rate of the bacteria, it is preferable to opt for disinfection of the enclosure since we can spend more time without intervening. Otherwise, it is better to eliminate infected individuals. Although this technique is applicable to any group of farm animals, we have shown an example by estimated values of the parameters inspired in the references considered. Two assumptions have been considered and applied to the two procedures. The different graphs and data collected in the shown tables and figures corroborate the conclusions presented here.

Author Contributions: Conceptualization, B.C., C.C., M.J.P., J.P. and E.S.; methodology, B.C., C.C., M.J.P., J.P. and E.S.; software, B.C., C.C., M.J.P., J.P. and E.S.; validation, B.C., C.C., M.J.P., J.P. and E.S.; formal analysis, B.C., C.C., M.J.P., J.P. and E.S.; investigation, B.C., C.C., M.J.P., J.P. and E.S.; writing-original draft preparation, B.C., C.C., M.J.P., J.P. and E.S.; writing-review and editing, B.C., C.C., M.J.P., J.P. and E.S. All authors have read and agreed to the published version of the manuscript.

Funding: This research received no external funding.

Institutional Review Board Statement: Not applicable.

Informed Consent Statement: Not applicable.

Data Availability Statement: Not applicable.

Conflicts of Interest: The authors declare no conflict of interest.

\section{Abbreviations}

The following abbreviations are used in this manuscript:

DFEP Disease-free equlibrium point

EEP Endemic equilibrium point

\section{References}

1. Buonomo, B.; Della Marca, R.; D'Onofrio, A. Optimal public health intervention in a behavioural vaccination model; the interplay between seasonality, behavior and latency period. Math. Med. Biol. 2019, 36, 297-324. [CrossRef]

2. Cantó, B.; Coll, C.; Sánchez, E. A study on vaccination models for a seasonal epidemic process. Appl. Math. Comput. 2014, 243, 152-160. [CrossRef]

3. Cao, H.; Zhou, Y. The discrete age-structured SEIT model with application to tuberculosis transmission in China. Math. Comput. Model. 2012, 55, 385-395. [CrossRef]

4. De la Sen, M.; Ibeas, A.; Alonso-Quesada, S.; Nistal, R. On the equilibrium points, boundedness and positivity of a SVEIRS epidemic model and constant constrained vaccination. Informatica 2011, 22, 339-370. [CrossRef]

5. Cantó, B.; Coll, C.; Sánchez, E. Estimation of parameters in a structured SIR model. Adv. Differ. Equ. 2017, 2017, 33. [CrossRef]

6. Cantó, B.; Coll, C.; Sánchez, E. Epidemic dynamics of an infection through patogen density in the environment. C. R. Acad. Bulg. Sci. 2016, 69, 835-844.

7. Cantó, B.; Coll, C.; Sánchez, E. Stabilization of an epidemic model via an N-periodic approach. Int. J. Appl. Math. Comput. Sci. 2018, 28, 185-195. [CrossRef]

8. Fitzgibbon, W.E.; Langlais, M.; Morgan, J.J. A mathematical model for indirectly transmitted diseases. Math. Biosci. 2004, 206, 233-248. [CrossRef] 
9. Joh, R.I.; Wang, H.; Weiss, H.; Weitz, J.S. Dynamics of indirectly transmitted infectious diseases with immunological threshold. Bull. Math. Biol. 2009, 71, 845-862. [CrossRef]

10. Ainseba, B.E.; Benosman, C.; Magal, P. A model for ovine brucellosis incorporating direct and indirect transmission. J. Biol. Dyn. 2010, 4, 2-11. [CrossRef]

11. Peter, O.J.; Ibrahim, M.O.; Oguntolu, F.A.; Akinduko, O.B.; Akinyemi, S.T. Direct and indirect transmission dynamics of Typhoid fever model by differential transform. Int. J. Sci. Technol. Educ. 2018, 6, 167-177.

12. Tien, J.H.; Earn, D.J.D. Multiple transmission Pathways and disease dynamics in a waterborne pathogen model. Bull. Math. Biol. 2010, 72, 1506-1533. [CrossRef]

13. Enatsu, Y.; Nakata, Y.; Muroya, Y. Global stability for a discrete sis epidemic model with immigration of infectives. J. Differ. Equ. Appl. 2012, 18, 1913-1924. [CrossRef]

14. Li, X.; Wang, W. A discrete epidemic model with stage structure. Chaos Solitons Fractals 2005, 263, 947-958. [CrossRef]

15. Nistal, R.; De la Sen, M.; Alonso-Quesada, S.; Ibeas, A. On a New Discrete SEIADR Model with Mixed Controls: Study of Its Properties. Mathematics 2019, 7, 18. [CrossRef]

16. Bittanti, S. Deterministic and Stochastic Linear Periodic Systems, in Time Series and Linear Systems; Springer: Berlin/Heidelberg, Germany, 1986.

17. Cantó, B.; Coll, C.; Pagán, M.J.; Poveda, J.; Sánchez, E. Spectral properties with application to epidemic models. Int. J. Complex Syst. Sci. 2016, 6, 1-7.

18. Aron, J.L. Mathematical modeling of immunity to malaria. Math. Biosci. 1988, 90, 385-396. [CrossRef]

19. Beaumont, C.; Brie, J.; Ducrot, A.; Zongo, P. Propagation of Salmonella within an industrial hen house. SIAM J. Appl. Math. 2012, 72, 1113-1148. [CrossRef]

20. Cao, H.; Tan, H. The discrete tuberculosis transmission model with treatment of latently infected individuals. Adv. Diff. Equ. 2015, 165, 1-18. [CrossRef]

21. González-Gunman, J.; Naulín, R. Analysis of a model of bovine brucellosis using singular perturbations. J. Math. Biol. 1994, 33, 211-223. [CrossRef]

22. Prévost, K.; Magal, P.; Beaumont, C. A model of Salmonella infection within industrial house hens. J. Theor. Biol. 2006, 242, 755-763. [CrossRef] [PubMed]

23. Vandeplast, S.; Dubois, R.; Beckers, Y.; Thonart, T.; Théwis, A. Salmonella in chicken: Current and developing strategies to reduce contamination at farm level. J. Food Prot. 2010, 73, 774-785. [CrossRef] [PubMed]

24. Zongo, P.; Viet, A.F.; Magal, P.; Beaumont, C. A spatio-temporal model to describe the spread of Salmonella within a laying flock. J. Theor. Biol. 2010, 267, 595-604. [CrossRef] [PubMed] 